\title{
Surface properties of AlInGaN/GaN heterostructure
}

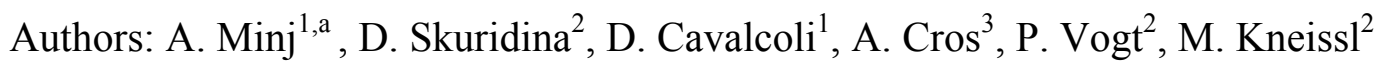
Affiliations:

1) Department of Physics and Astronomy, University of Bologna, Bologna, Italy

2) Institute of Materials Science (ICMUV), University of Valencia, P.O. Box 22085, ES-46071, Valencia, Spain

3) Institut für Festkörperphysik, Technische Universität Berlin, Hardenbergstraße 36, 10623 Berlin, Germany

a) Present address: CIMAP UMR 6252 ENSICAEN, 6 Bd Maréchal Juin 14050, Caen, France

\begin{abstract}
Surface structural, electronic and electrical properties of the quaternary alloy AlGaInN/GaN heterostructures are investigated. Surface termination, atomic arrangement, electronic and electrical properties of the (0001) surface and (10-11) Vdefect facets have been experimentally analyzed using various surface sensitive techniques including spectroscopy and microscopy. Moreover, the effect of sub-band gap (of the barrier layer) illumination on contact potential difference (VCPD) and the role of oxygen chemisorption have been studied.
\end{abstract}




\section{Introduction}

Due to strong polarization fields, III-Nitride heterostructures such as $\mathrm{AlGaN} / \mathrm{GaN}$ are inherently advantageous for the formation of two-dimensional electron gas (2DEG) without the need of intentional doping as in the case of $\mathrm{AlGaAs} / \mathrm{GaAs}$. For $\mathrm{Al}_{\mathrm{x}} \mathrm{Ga}_{1}$ ${ }_{\mathrm{x}} \mathrm{N} / \mathrm{GaN}$ heterostrucutres, as the Al-content is increased, 2DEG density could be tuned from $10^{12}$ to $10^{13} \mathrm{~cm}^{-2}$, and an electron mobility varying over 1200 to $2000 \mathrm{~cm}^{2} / \mathrm{V}$-s at room temperature has been reported $[1,2]$. This allows for the realization of high quality high electron mobility transistors (HEMT) suitable for high frequency applications [3, 4]. Despite the tenability of $\mathrm{AlGaN}$, incorporation of more $\mathrm{Al}$ into $\mathrm{AlGaN}$ matrix leads to strong lattice mismatch with GaN and high tensile strain leading to the formation of cracks or misfit dislocations $[5,6]$. Thus, alternatively to $\mathrm{AlGaN} / \mathrm{GaN}$ heterostructure, quaternary alloy of AlGaInN could be used as the barrier layer as it allows independent tuning of lattice constant and the band gap. Additionally, due to stronger polarization constants, for the same lattice strain as in $\mathrm{AlGaN} / \mathrm{GaN}$, a higher 2DEG concentration could be achieved [7-10]. This heterostructure originally developed for high-speed devices, has recently experienced a renewed interest due to its wide and tunable band gap, strong polarization and very high sensitivity of 2DEG concentration to the surface barrier that makes it feasible to be used in reduction based applications [11], solar blind UV detectors $[12,13]$ and molecule detectors [14-16] is an interest of scientific research. In this report, surface atomic and electronic structure is experimentally determined from different surface sensitive techniques and the role of V-defects is discussed. And the 
process of oxygen chemisorption on surface photovoltage is assessed with aid of UV illumination.

\section{Experiment}

$\mathrm{AlGaInN}(15 \mathrm{~nm}) / \mathrm{AlN}(2.5 \mathrm{~nm}) / \mathrm{GaN}$ heterostructures were grown along (0001) on $\mathrm{GaN}(3$ $\mu \mathrm{m}) /$ sapphire templates by metal organic chemical vapor deposition (MOCVD) AIXTRON system. Rutherford backscattering spectroscopy analysis by Brazzini et al determined the composition of the layer to $57 \% \mathrm{Al}, 36 \% \mathrm{Ga}$ and $7 \%$ In $[17,18]$. In the present study, besides assessment of surface composition, valence band energy was also determined by x-ray photo-spectroscopy (XPS) in ultra-high vacuum at room temperature. In the XPS setup, the kinetic energy (K.E.) of the electrons ejected of the atomic energy levels was analyzed by the spectrometer using a hemispherical sector analyzer (HSA) combined with a detection system. The X-ray source providing Al-K $\alpha$ radiations with line energy of $1486.6 \mathrm{eV}$ was used and the total instrumental resolution determined from the FWHM of the Fermi edge was less than $400 \mathrm{meV}$ [19]. To reduce Cand $\mathrm{O}$ - impurities, annealing at $500{ }^{\circ} \mathrm{C}$ and $650{ }^{\circ} \mathrm{C}$ for 10 minutes were also performed prior to measurements. The analyzed samples were then transferred to the scanning tunneling microscopy (STM) UHV chamber to analyze the density of states. Topography and surface potential were also mapped simultaneously under ambient conditions with AFM. With the first lock-in amplifier set to the resonance frequency of the cantilever, phase-locked loop (PLL) control system modulating the excitation frequency to keep the phase constant, AC bias of $1 \mathrm{~V}(7 \mathrm{kHz})$ and a DC variable applied to the AFM tip, and $2^{\text {nd }}$ lock-in amplifier set at $7 \mathrm{kHz}$ measuring the electrostatic force gradient (in-phase component from the $1^{\text {st }}$ lock-in), KPFM maps were obtained by nullifying the 
electrostatic signal with the DC variable. The tip-sample distance is assessed to be around $10 \mathrm{~nm}$. Before the AFM measurements, samples were de-greased by standard chemical preparation and native oxide was etched in boiling aqua regia. An additional setup consisting of fiber-coupled-100W mercury lamp to the sample under the AFM tip was used to perform UV Illumination. The total output power measured was $\sim 40 \mathrm{~mW}$.

\section{Results and discussions}

XPS spectra of the sample were obtained for varying photoemission angles $\left(\theta=0^{\circ}\right.$ to $80^{\circ}$ ) i.e. the angle formed between the direction normal to the surface and the detector. For $\theta=80$ a depth of $\sim 2 \mathrm{~nm}$ was obtained, allowing surface sensitive measurements. Based on the composition determined from the Ga3s, A12s, In $3 \mathrm{~d} / 2$ and N1s core levels, as shown in fig. 1a for various photoemission angles, it is assessed that the surface is Alterminated. The surface diffraction pattern (1b) obtained through LEED with energy beams between $25 \mathrm{eV}$ and $225 \mathrm{eV}$ incident normally to the surface shows that the surface is $(1 \mathrm{x} 1)$. As can be seen from figures $1 \mathrm{a}$ and $1 \mathrm{~b}$, the annealing up to $650{ }^{\circ} \mathrm{C}$ had no effect on bulk composition, surface termination and the surface reconstruction. However, the composition is not homogeneous across the investigated depth, but $\mathrm{N}$-content reduces and Al content increases the surface. For an Al-terminated surface and $\mathrm{N}$ in the sub-surface layer, the expected $\mathrm{Al} / \mathrm{N}$ ratio should be close to $1: 1$, however, the measured $\mathrm{N}$-content is $\sim 15$ to $25 \%$. The increased $\mathrm{Al} / \mathrm{N}$ could suggest a presence of an Al-adlayer. Figure 2 shows the A12s spectra for different photoemission angles $0^{\circ}, 60^{\circ}$ and $80^{\circ}$. Though, the binding energy (B.E.) of the core level at $0^{\circ}$ and $60^{\circ}$ are same corresponding to $\mathrm{Al}-\mathrm{N}$, the shift of the B.E. towards the higher energy at $80^{\circ}$ corresponds to Al-O, ascertaining the presence of an Al-adlayer [20]. 

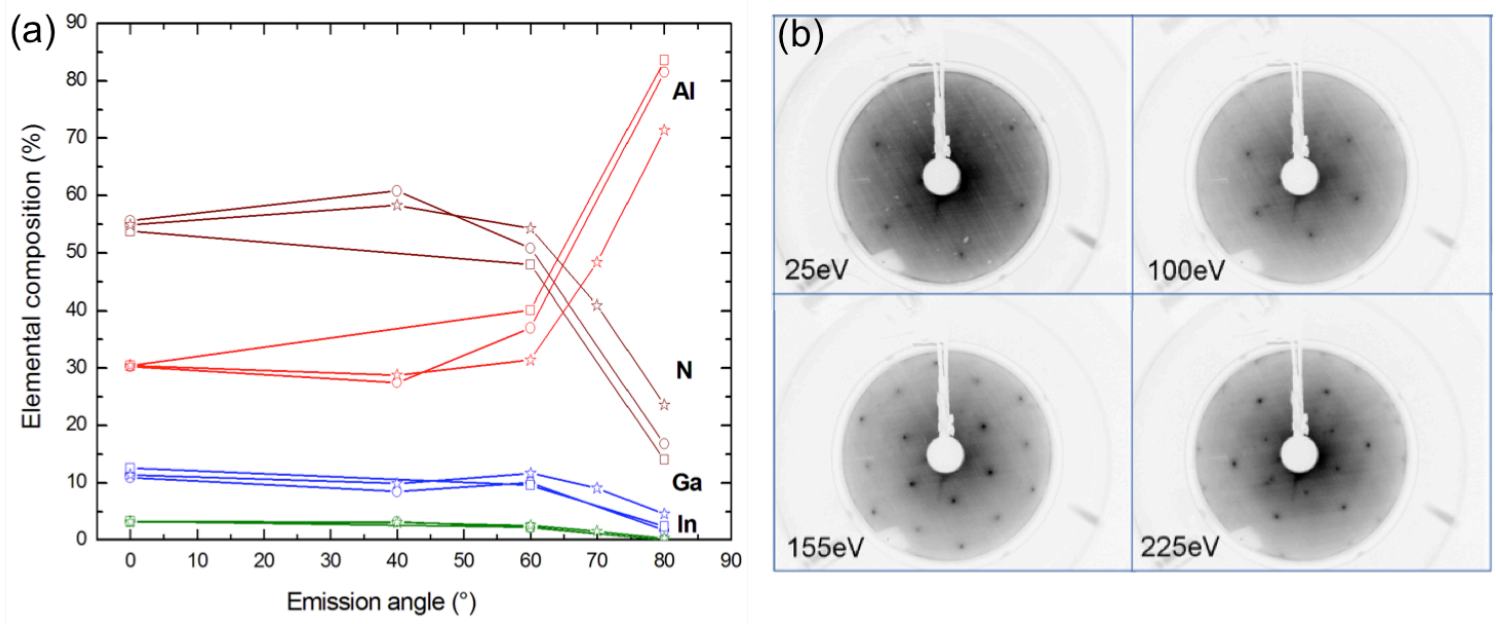

Figure 1 (a) Estimated atomic percentage of $\mathrm{In}, \mathrm{Ga}, \mathrm{Al}$ and $\mathrm{N}$ at different photoemission angles for different annealing temperatures, $25^{\circ} \mathrm{C}$ (in squares), circle $500{ }^{\circ} \mathrm{C}$ (in circles) and $650{ }^{\circ} \mathrm{C}$ (in stars). (b) Inverse LEED images showing $1 \mathrm{x} 1$ surface reconstruction at different incident electron beam energies.

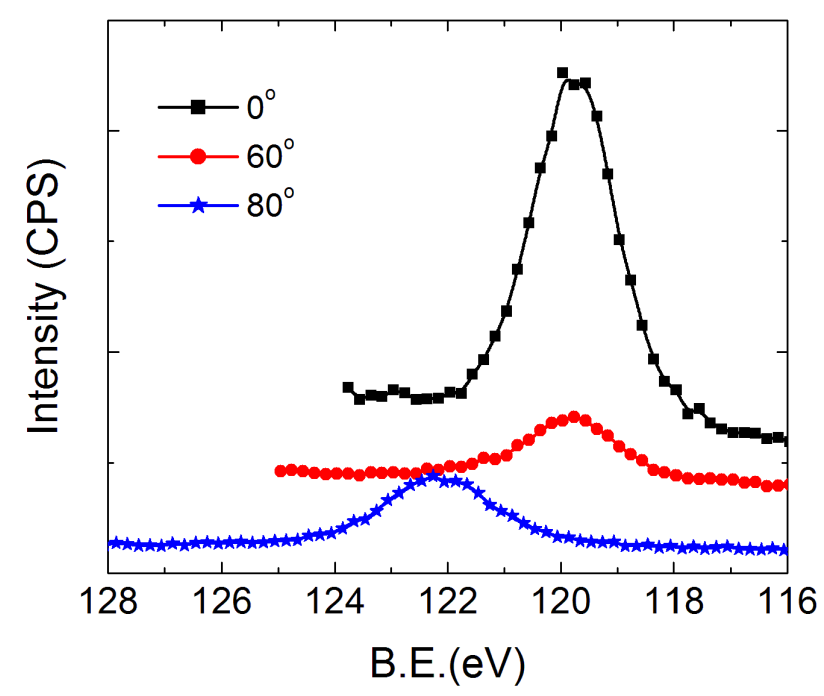

Figure $2 \mathrm{Al} 2 \mathrm{~s}$ core level spectra for different photoemission angles.

The valence band of the quaternary alloy with respect to the Fermi-level is determined from the XPS spectra and is shown in figure 3. The Fermi-level $\mathrm{E}_{\mathrm{F}}$ corresponds to a B.E. of $0 \mathrm{eV}$. These measurements were carried out on the as-grown sample and after the sample was annealing at $500{ }^{\circ} \mathrm{C}$ and $650{ }^{\circ} \mathrm{C} . \mathrm{E}_{\mathrm{F}}-\mathrm{E}_{\mathrm{V}}$ in the un-annealed sample was 
around $3.53 \mathrm{eV}$, which is reduced to $3.23 \mathrm{eV}$ and $3.08 \mathrm{eV}$ after annealing at $500{ }^{\circ} \mathrm{C}$ and $650{ }^{\circ} \mathrm{C}$, respectively. From the estimated bandgap $\mathrm{E}_{\mathrm{G}}$ of around $4.55+/-0.07 \mathrm{eV}$ [21], the Fermi-level is assessed to be at $1.02 \mathrm{eV}$ from the conduction band for the un-annealed sample. With increasing annealing temperatures, as $\mathrm{O}$ and $\mathrm{C}$-impurities desorbs from the surface, $\mathrm{E}_{\mathrm{C}}-\mathrm{E}_{\mathrm{F}}$ increases to $1.32 \mathrm{eV}$ and further to $1.47 \mathrm{eV}$. This behavior is consistent with the shift of the $E_{F}$ with the annealing of $\mathrm{GaN}$ substrates due to desorption of Oxygen, which is identified as donors [22].

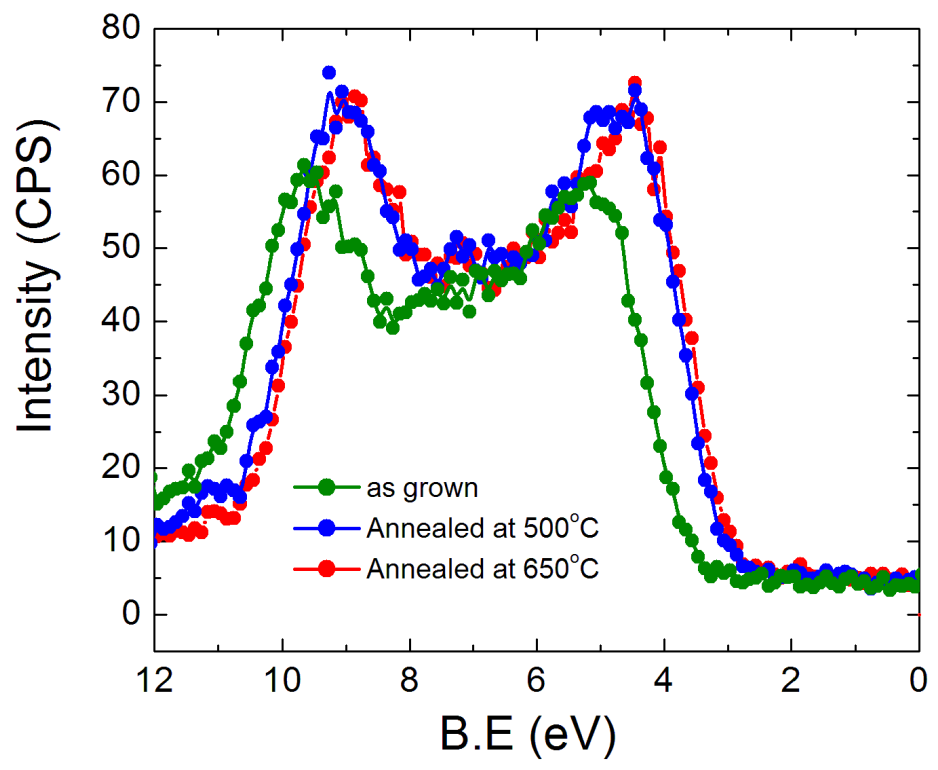

Figure 3: AlInGaN XPS spectra at the valence band of the as-grown and annealed samples $\left(500{ }^{\circ} \mathrm{C}, 650{ }^{\circ} \mathrm{C}\right)$.

Surface structural properties were assessed also by STM. As shown in the AFM micrograph in figure 4a, the surface morphology consists of granular structures of size $200 \mathrm{~nm}$ in diameter and V-defects with density of $3 \times 10^{8} \mathrm{~cm}^{-2}$. This is comparable to the density of threading dislocations in GaN substrate used. The rms roughness calculated on an area of $2 \times 2 \mu \mathrm{m}$ is around $0.26 \mathrm{~nm}$. Most of the granular structures are formed around 
the V-defects. This is similar to what has been observed around screw-type TDs for MBE growth of GaN $[23,24]$. V-defects are already known to be detrimental to the AlGaN or AlInN based devices as they can cause severe leakage due to metallic atom decoration or electrically active defect levels allowing hopping mechanism in reverse bias [25]. Apart from V-defects, surface roughness and surface correlation length that can vary over different growth conditions are speculated to limit the electron mobility in the HEMT devices due by remote surface roughness (RSR) mechanism [26]. Thus, as shown in figure 4, along with micro-structural properties from AFM, nano-structural details with atomic resolution were also obtained using STM. In STM scan area (fig. 4b), it can be seen that the surface is composed of well-aligned nanosize growth domains and possibly they are formed along the underlying step-terrace of $\mathrm{GaN}$ substrate. Atomic features could be resolved on the growth-domains, showing surface atomic arrangement corresponding to (1x1) surface reconstruction (inset of fig. 4b). This is in confirmation with the LEED patterns. The typical roughness calculated from AFM scans over $2 \times 2 \mu \mathrm{m}$ or from STM scans over $50 \times 50 \mathrm{~nm}^{2}$ is around $0.25 \mathrm{~nm}$, which is equivalent to half the unit cell of GaN. However, the height-height correlation lengths for the two are significantly different. The correlation lengths calculated from AFM scan was around 55 $\mathrm{nm}$ and from STM scan was around $2 \mathrm{~nm}$. For the given roughnesses, the 2DEG mobility estimated from the two correlation lengths values differ each other by at least three orders; $>10^{6} \mathrm{~cm}^{2} . \mathrm{V}^{-1} \mathrm{~s}^{-1}$ for $55 \mathrm{~nm}$ and $\sim 10^{3} \mathrm{~cm}^{2} . \mathrm{V}^{-1} \mathrm{~s}^{-1}$ for $2 \mathrm{~nm}$, which is in agreement with the experimental value of $\sim 1290 \mathrm{~cm}^{2} \mathrm{~V}^{-1} \mathrm{~s}^{-1}$ at $300 \mathrm{~K}$ published elsewhere [26]. 


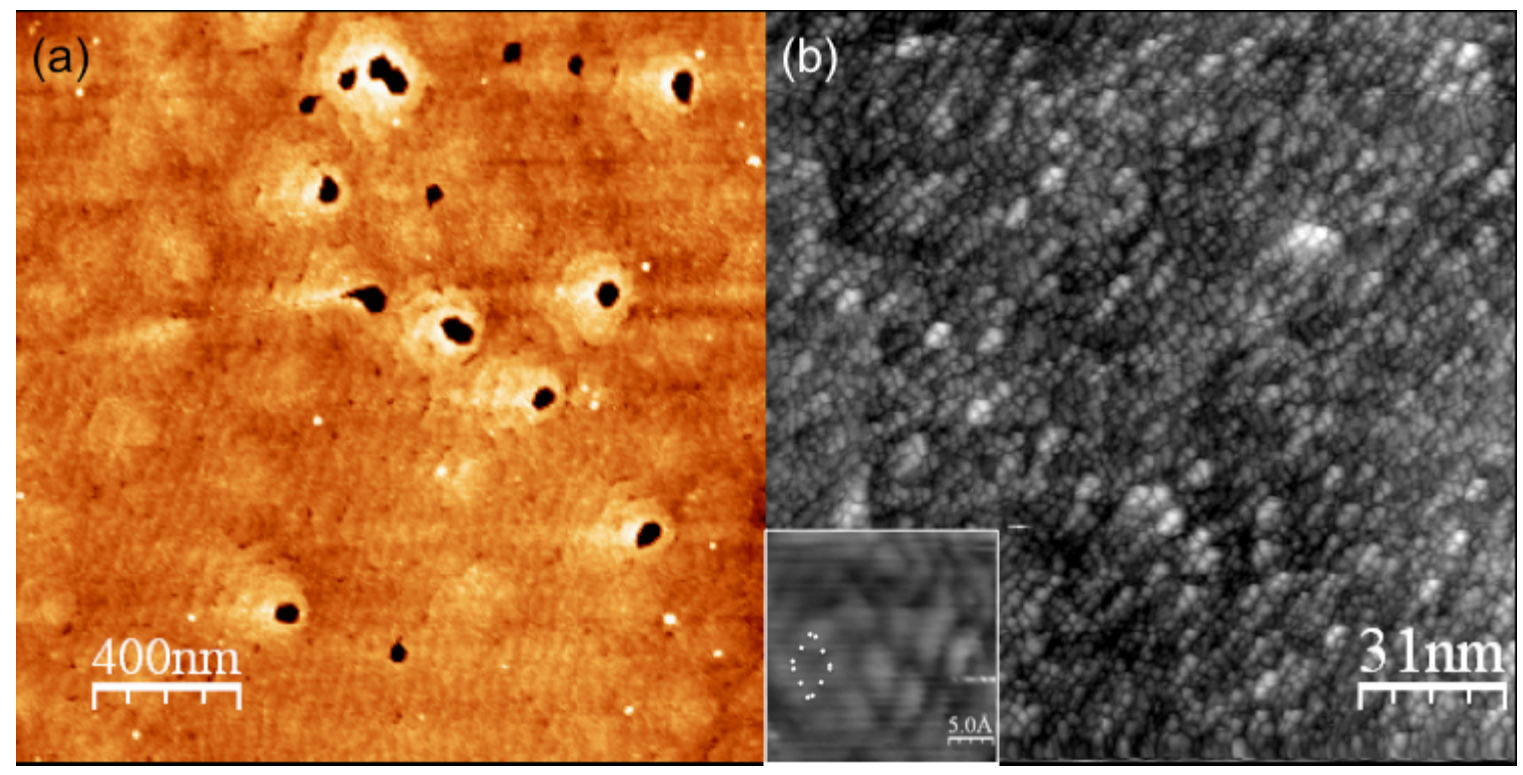

Figure 4: (a) AFM (1 $\mu \mathrm{m} \times 1 \mu \mathrm{m})$ and (b) STM (100 nm x $100 \mathrm{~nm})$ topography in constant-current mode of $\mathrm{AlInGaN/GaN}$ heterostructure. Inset shows the atomic arrangement corresponding to (1x1) unit cell.

For the observation of the density of states, local Scanning tunneling spectroscopy (STS) was performed on V-defects and on the defect free region for comparison. In STS, current-voltage (I-V) behavior is due to the tunneling of electrons from occupied states to unoccupied states i.e. the tunneling current involving electronic states outside the forbidden gap occurs for the two conditions: when $+\mathrm{eV}>\mathrm{E}_{\mathrm{F}, \mathrm{m}}-\mathrm{E}_{\mathrm{CBM}}$ and $-\mathrm{eV}>\mathrm{E}_{\mathrm{VBM}^{-}}$ $\mathrm{E}_{\mathrm{F}, \mathrm{m}}$, where EFM, ECBM and EVBM are the Fermi level, conduction band minimum and valence band maximum, respectively. However, for tunneling biases corresponding to energies within the energy gap, observation of current could occur if (i) intrinsic/extrinsic surface states exist in the band gap and/or (ii) defects are present at the surface. Figure 5a shows the $300 \times 300 \mathrm{~nm} \times \mathrm{nm}$ STM image. It comprises three V-defects marked as $\underline{1,2}$ and 3 (in dashed box). STS measurements were performed along the line starting from a free surface to the V-defect in order to observe the existence of difference in density of 
states in two different regions. Normalized differential conductivity (dI/dV)/(I/V) extracted from I-V measurements against the sample bias are shown in fig. $5 \mathrm{~b}$. Average of the $(\mathrm{dI} / \mathrm{dV}) /(\mathrm{I} / \mathrm{V})$ on regions outside $\mathrm{V}$-defects (in black curve) indicates the maxima of the valence band around $2 \mathrm{eV}$, while at $\mathrm{V}$-defects, this is observed at $\mathrm{E}_{\mathrm{VBM}}-0.64$. The shift in the occupied states towards the Fermi level by $0.64 \mathrm{~V}$ at $\mathrm{V}$-defect could arise from the semi-polar nature $\{10-11\}$ of the facets, which could have a different surface reconstruction and surface termination than the $\mathrm{c}$ surface. For instance, the $\mathrm{N}$-termination of $\{10-11\}$ surfaces of GaN is energetically favorable and has high affinity to oxygen $[27,28]$.
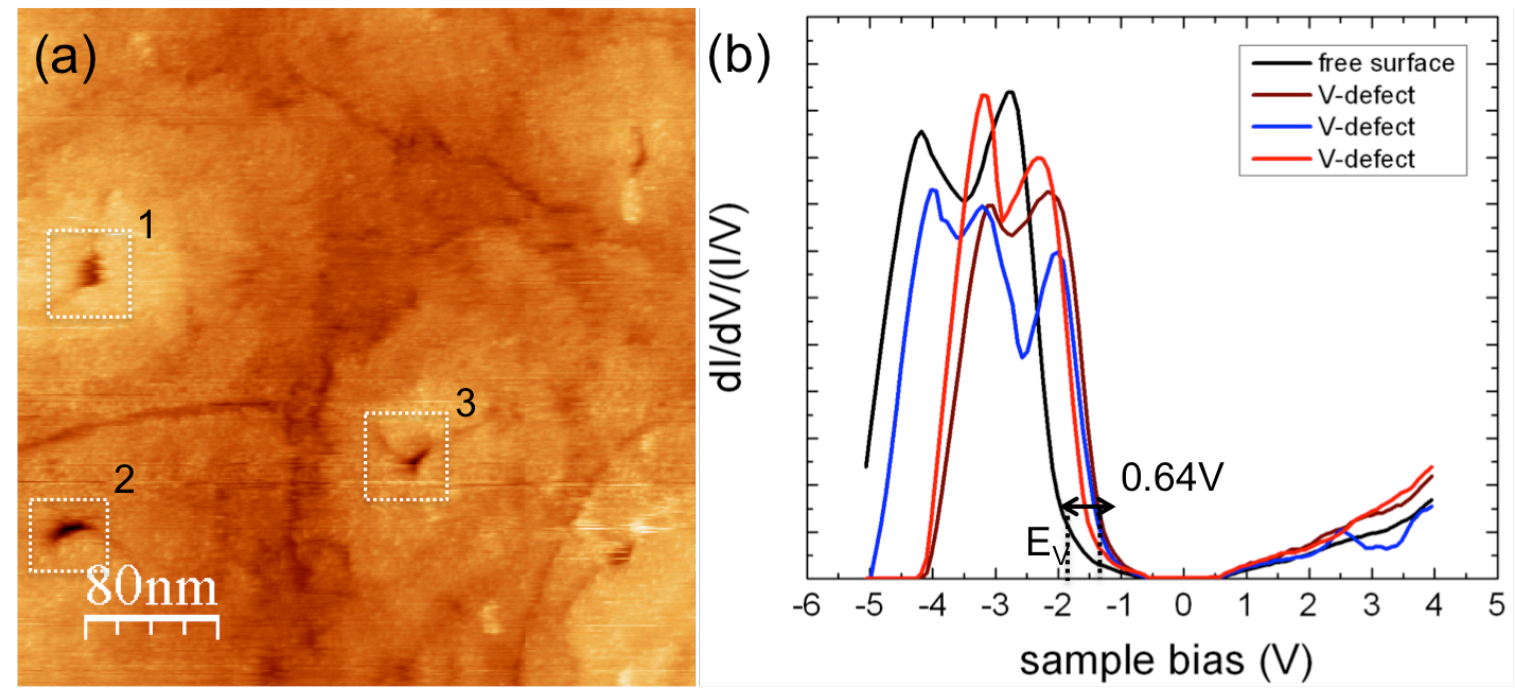

Figure 5: (a) shows STM image of $250 \mathrm{~nm} \times 250 \mathrm{~nm}$ area. V-defects are marked in dashed box. (b) Average (dI/dV)/(I/V) against bias for region surrounding V-defect (in black) and local (dI/dV)/(I/V) over V-defects marked in dashed boxes 1, 2 and 3 (red, blue, brown curves) are shown.

Figure 6(a), shows the $2 \mu \mathrm{m} \times 2 \mu \mathrm{m}$ topography. The roughness measured on this area is around $0.25 \mathrm{~nm}$. As mentioned earlier, step terraces, most likely originating from the GaN surface in convolution with granular features can be observed. Since this density of V-defects is of the same order of TDD present in $\mathrm{GaN}$ template used for the growth, it 
could be assumed that most of these V-defects are related to TDs. As KPFM maps are highly prone to noise, either from the electronics or from the noise of the laser diode used in the optical system of the AFM, fig. 6(b) has been processed by fast Fourier Transform (FFT) filter. Corresponding to this topography, KPFM map is shown in fig. 6(b). It is important to note that, since information on VCPD is obtained when the electrostatic signal strength is 0 , KPFM maps are highly prone to noise, either from the electronics and/or from the noise of the laser diode used in the optical system of the AFM. Thus, the data of fig. 6(b) shown here has been processed by an FFT filter. From the comparison with the topography, it can be seen that at the V-defects there is a reduction in the VCPD by $\sim 0.2 \mathrm{~V}$, which indicates an equivalent increment in the work function. Height profile and VCPD profile across two V-defects is shown in fig 6(c). Here, VCPD profile from the as-obtained data along with the FFT filtered map is included to show correlation and it is clear that the FFT filtered data describes reasonably well the raw data. At the Vdefects, it can be seen that the VCPD reduction is seen strictly inside the V-defect. This is an indication that the increment in work function is not due to fixed negative charges at this defect. If it would be the case, a smooth tail of the VCPD spreading out to the planar surface from the core of $\mathrm{V}$-defect would be observed, equivalent to the potential changes around a line charge [29]. In order to verify that VCPD contrast arising on the V-defect is not induced from the tip-shape artifact, which could cause reduction of the electrostatic signal to 0 (or equivalently affecting VCPD signal), electrostatic force gradient microscopy (EFGM) map of the area of interest (fig. 6d) is shown in fig. 6e and EFGM profiles across several V-defects are presented in fig 6f. It is clear that the EFGM signal is only improved at the V-defect, thus, contradicting the idea of the tip-sample artifact. 
The difference in work function for the two facets could be attributed to either difference in the intrinsic electronic properties of (0001) plane and (1-101) planes or due to the presence of an oxide. Due to lack of a general consensus of the value of electron affinities for the c-plane (varying between 2.7 and $4.1 \mathrm{eV} \mathrm{[30])} \mathrm{and} \mathrm{the} \mathrm{available} \mathrm{values} \mathrm{for} \mathrm{m-}$ plane (assumed to be $4.1 \mathrm{eV}$ ), the former case cannot be verified. However, in the latter case, V-defects could play an important role in defining the electron affinity of the surface. As shown by Mishra et al. [31], V-defects assist in strong oxygen chemisorption at the surfaces of $\mathrm{AlGaN} / \mathrm{GaN}$ heterostructures, which in turn, increases the electron affinity by magnitude ranging from 0.2 to $0.6 \mathrm{eV}$. For this case, since chemisorption of oxygen can preferentially occur at the V-defect facets, where enhancement of electron affinity is expected. As explained earlier in the STS analysis on V-defect, a shift in the VBM towards the Fermi-level by $0.64 \mathrm{eV}$ is observed that could also yield an increment in work function cannot be ruled out.
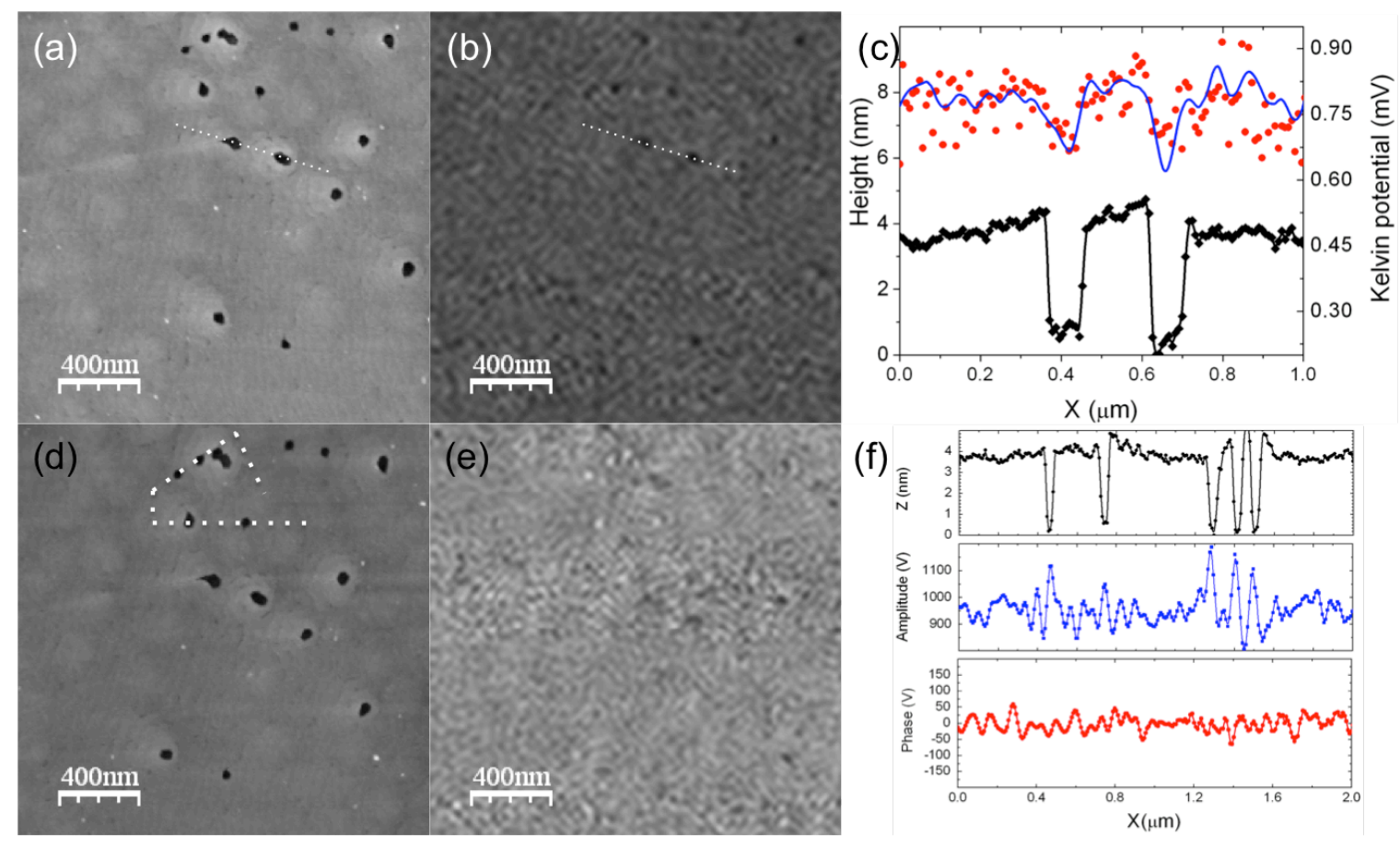
Figure 6: (a) Topography and its corresponding (b) KPFM map (after FFT filter). Height, VCPD profiles with (lines) and without (symbols) FFT filter are shown in (c). (d) Topography and its corresponding (e) map of amplitude of the out-of-phase component at $7 \mathrm{kHz}$. Height, A2nd dynamic and P2nd dynamic profiles are shown in (f).

Surface photovoltage (SPV) study was performed using Kelvin probe force microscopy by measuring VCPD under dark and UV illumination. Detailed reports on the surface photovoltage spectroscopy on the heterostructures are published elsewhere [3234]. Figure 7a shows the time evolution of the SPV averaged over each line along the fast-scan axis of the scan area. After the dark measurements, the surface is illuminated with UV light at around $1.5 \mathrm{~min}$ (marked as blue line in the graph) and an abrupt change in the SPV by $\sim 0.1 \mathrm{~V}$, which continues to increase is observed. The positive SPV is in agreement with the observations made by Pandey et al. on the same set of samples and similar behavior has also been observed in other heterostructures with 2DEG [35]. In the absence of above-band gap absorption of photons (the emission lines from the Mercuryarc lamp are below the band gap of the barrier layer), this SPV could not be attributed to the barrier layer. However, because of the ground state energy level $\mathrm{E}_{0}$ lying below the $\mathrm{E}_{\mathrm{F}}$ and $1^{\text {st }}$ excited state $E_{1}$ lying close to the $E_{F}$ in the triangular quantum well of the $2 \mathrm{DEG}$, electronic transitions from (1) valence band of the barrier layer, (2) defect levels at the interface or in the (3) barrier layer could occur, leaving positive holes in the valence band and positive charge in the defect levels. The holes formed in the barrier layer, would move towards the surface due to strong field in the barrier layer, forming a net positive charge at the surface (schematically shown in fig. 7b). From the VCPD measurements shown here, the first case is more likely, as SPV continues to develop with time in the scale of seconds to minutes, which is an indication of accumulation of positive charge at 
surface states with slow carrier dynamics, and slow states are well known to be formed at oxide surfaces (AlOx in this case).
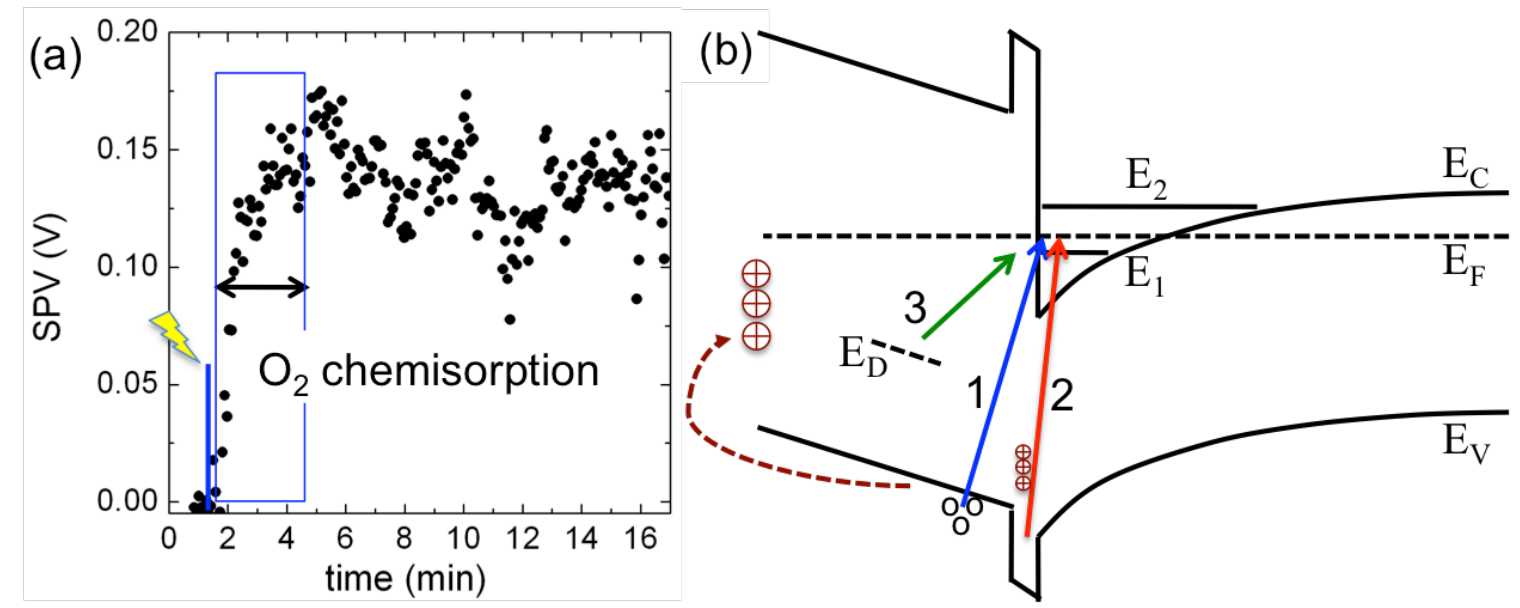

Figure 7: (a) SPV as a function of time. UV is switched on at 0.2 (b) Possible absorption processes responsible for positive SPV.

\section{Conclusion}

Quaternary alloy AlInGaN/GaN heterostructures were probed with surface sensitive techniques including XPS, LEED, STM and Kelvin probe force microscopy. The surface barrier height (Ec-Ef) was determined to be $\sim 1.02 \mathrm{eV}$ for as-grown sample but shift to a higher value by $0.45 \mathrm{eV}$ with desorption of oxygen from the surface after annealing at $650{ }^{\circ} \mathrm{C}$. The surface corresponds to $(1 \times 1)$ and is Al-terminated with the presence of Aladlayer. At the facets of the V-defects, possibly $\{1-101\}$, an increment in the workfunction by $\sim 0.2 \mathrm{eV}$ is observed, which could be either related to preferential oxygen chemisorption at the facets and/or due to the shift of the VBM by $0.64 \mathrm{eV}$ as assessed with STM. With UV illumination-coupled KPFM, a positive surface SPV is observed which is understood in terms of absorption of e-h pairs from the valence band of the barrier layer to the discrete energy levels of the $2 \mathrm{DEG}$ region.

\section{Acknowledgements}


We are very thankful to Prof. A. Cavallini for useful discussion and we gratefully acknowledge Dr. H. Behmenburg, Dr. C. Giesen, and Prof. M. Heuken of Aixtron SE,

Herzogenrath, Germany, for sample growth. This work was supported by the European Commission under Project no. PITN-GA-2008 213238-RAINBOW.

\section{References}

$1 \quad$ Smorchkova, J. Appl. Phys., 86, 4520 (1999)

$2 \quad$ Gaska Appl. Phys. Lett., 74287 (1999)

3 U.K. Mishra Proceedings of the IEEE 90, Issue 6, 1022 - 1031 (2002)

4 Ambacher J. Appl. Phys., Vol. 85, No. 6, 15 March (1999)

$5 \quad$ Floro J. Appl. Phys., 967088 (2004),

$6 \quad$ Hearne Appl. Phys. Lett., 76, 1534 (2000)

7 Ketteniss, Semicond. Sci. Technol. 25, 075013 (2010)

$8 \quad$ Khan Applied Physics Letters 75, 2806 (1999)

$9 \quad$ Liu Applied Physics Letters 86, 223510 (2005)

10 Hahn Semicond. Sci. Technol. 27, 055004 (2012)

11 Yotsuhashi Appl. Phys. Lett. 100, 243904 (2012)

12 Biyikli, Appl. Phys. Lett., Vol. 81, 3273 (2002)

13 Parish, Appl. Phys. Lett. 75, 247 (1999)

14 Wang Appl. Phys. Lett. 91, 042114 (2007)

15 Kang J. Appl. Phys. 104, 031101 (2008)

16 Kang, Appl. Phys. Lett. 91, 112106 (2007)

17 Brazzini Semicond. Sci. Technol. 28, 055007 (2013)

18 Gunasekar AIP Advances 4, 127101 (2014)

19 D. Skuridina, D. V. Dinh, B. Lacroix, P. Ruterana, M. Hoffmann, Z. Sitar, M. Pristovsek, M. Kneissl and P. Vogt, J. Appl. Phys. 114, 173503 (2013)

20 Duo Cao, Xinhong Cheng, Ya-Hong Xie, Li Zheng, Zhongjian Wang, Xinke Yu, Jia Wang, Dashen Shen and Yuehui Yu, RSC Adv., 5, 37881-37886 (2015)

21 Bokov, Semicond. Sci. Technol. 30, 085014 (2015) 
22 Jang, H. W., Baik, J. M., Lee, M. K., Shin, H. J., \& Lee, J. L., Journal of The Electrochemical Society, 151(8), G536-G540 (2004)

23 Qian, Appl. Phys. Lett., 67, 2284 (1995)

24 Heying, J. Appl. Phys., 85, 6470 (1999)

25 Eller, J. Vac. Sci. Technol. A, 31, 050807 (2013)

26 Pandey Acta Materialia 60 (6), 3176-3180

27 Grossner, phys. stat. sol. (b), 216, 675 (1999)

28 Rosa, Physical Review B, 73, 205346 (2006)

29 Simpkins, J. Appl. Phys., 91, 9924 (2002)

30 M. Motyka, M. Syperek, R. Kudrawiec, J. Misiewicz, M. Rudziński, P. R. Hageman and P. K. Larsen, Appl. Phys. Lett. 89, 231912 (2006)

31 Mishra Phys.Chem., 17, 15201 (2015)

32 Pandey, J. Appl. Phys. 112, 123721 (2012)

33 Cavalcoli Appl. Phy. Lett. 98, 142111 (2011)

34 Surface and Defect States in Semiconductors Investigated by Surface Photovoltage D Cavalcoli, B Fraboni, A Cavallini - Semiconductors and Semimetals, (2015)

35 Kim, Sci Rep. 5, 8531 (2015), Solodky, Appl. Phys. Lett., 83, 2465 (2003) 